\title{
Le caballito de totora à Huanchaco, une embarcation singulière et une pratique de pêche millénaire au Pérou
}

El caballito de totora en Huanchaco, una embarcación singular de una práctica de pesca milenaria en el Perú

The caballito de totora in Huanchaco, a singular boat of a thousand-year-old fishing practice in Peru

\section{Marina Quiñe}

\section{CpenEdition \\ Journals}

Édition électronique

URL : https://journals.openedition.org/ethnoecologie/6427

DOI : 10.4000/ethnoecologie.6427

ISSN : 2267-2419

Éditeur

Laboratoire Éco-anthropologie

\section{Référence électronique}

Marina Quiñe, «Le caballito de totora à Huanchaco, une embarcation singulière et une pratique de pêche millénaire au Pérou », Revue d'ethnoécologie [En ligne], 19 | 2021, mis en ligne le 30 juin 2021, consulté le 17 décembre 2021. URL : http://journals.openedition.org/ethnoecologie/6427 ; DOI : https://doi.org/10.4000/ethnoecologie.6427

Ce document a été généré automatiquement le 17 décembre 2021.

Revue d'ethnoécologie est mis à disposition selon les termes de la licence Creative Commons Attribution - Pas d'Utilisation Commerciale - Pas de Modification 4.0 International. 


\title{
Le caballito de totora à Huanchaco, une embarcation singulière et une pratique de pêche millénaire au Pérou
}

\author{
El caballito de totora en Huanchaco, una embarcación singular de una práctica \\ de pesca milenaria en el Perú \\ The caballito de totora in Huanchaco, a singular boat of a thousand-year-old \\ fishing practice in Peru
}

Marina Quiñe

\section{NOTE DE L'AUTEUR}

J'ai été chargée par le Muséum national d'Histoire naturelle d'acquérir un caballito de totora, afin de le faire venir en France. J'ai donc commandé un caballito à M. Fidel Huamanchumo, pêcheur à Huanchaco, qui s'est mis à l'ouvrage et m'a permis d'en faire un reportage. Ce caballito fait partie aujourd'hui de l'exposition permanente du Musée de l'Homme à Paris, depuis sa réouverture en 2015, après avoir traversé l'Atlantique, mais dans la cale d'un cargo!

\section{Aspects généraux}

1 Au Pérou, la pêche représente une activité pratiquée depuis plus de dix mille ans, commençant avec les premières communautés sédentaires côtières qui vivaient de façon presque exclusive de la mer, jusqu'à nos jours, où la pêche n'a pas perdu son importance (Rostworowski 2005) ${ }^{1}$. 
2 Dans l'écosystème marin péruvien (de Humboldt ou courant de Humboldt), la pêche est la plus importante activité économique pratiquée. Par exemple, la valeur des produits exportés issus de la pêche au Pérou entre 1998 et 2010 (farine et huile de poisson et produits de consommation humaine directe), a atteint 19723100000 US\$ représentant 98158662 tonnes de matière première extraite. (Majluf 2006, Revista Pesca 2011, Perú Ministerio de la Producción 2015).

3 Le Pérou est le deuxième pays le plus important du monde au niveau des volumes de pêche annuellement rapportés, après la Chine, et il est le premier pays exportateur de farine et d'huile de poisson (d'anchois péruviens Engraulis ringens). La pêche industrielle est devenue depuis les années 60 la deuxième activité économique la plus rentable (après l'industrie minière) générant des bénéfices sous forme de devises pour le pays, et d'énormes revenus pour les industriels qui font partie de la Société Nationale des Pêcheries (Majluf 2006).

4 À la différence de la pêche industrielle, la pêche artisanale est une activité qui s'exerce principalement dans un cadre d'économie familiale, de subsistance, qui dépend de la mer et de ses ressources, du souci et de la responsabilité de la famille et de la communauté (García 2001).

On va s'intéresser à une pratique de pêche ancestrale qui perdure jusqu'à nos jours sans variations significatives : la pêche à bord du caballito de totora, et plus particulièrement à l'embarcation utilisée à cette fin. La zone d'étude retenue a été la communauté de pêcheurs de Huanchaco (La Libertad, nord du Pérou). Cette communauté a une continuité historique qui remonte à plus de mille ans, une activité continue dans le temps d'une pratique de pêche, unique au monde. Ils continuent à transmettre cet héritage de génération en génération malgré une problématique complexe actuelle, qui ne sera pas le sujet de ce texte.

\section{Huanchaco, berceau du caballito de totora}

6 La municipalité de Huanchaco se situe dans le département de La Libertad sur la côte nord du Pérou (0804'34" LS - 7906’57" LO). Elle a une surface de $333,9 \mathrm{~km}^{2}$. Elle se trouve sur la rive droite de la vallée du fleuve Moche (principale source en eau potable de la zone) à 12,6 km au nord-est de la ville de Trujillo (Capitale du département) et à $560 \mathrm{~km}$ de Lima.

7 L'écosystème marin côtier de Huanchaco est constitué d'eaux froides, typiques du courant de Humboldt, avec une plateforme continentale de grande extension qui bénéficie de l'abondance des ressources pour les poissons.

8 Le fond marin côtier est de type sableux - rocheux, sédiment optimum pour l'établissement des algues. On a un écosystème de prairie de macro-algues rouges de taille moyenne, dont l'espèce la plus répandue est Chondracanthus chamissoi « mococho » « cochayuyo » ou « yuyo » en langue vernaculaire (Carbajal et al. 2005).

9 Par ailleurs, l'activité de la pêche industrielle en face de la côte de Huanchaco (au delà de 5 milles marins ${ }^{2}$ ) est assez intense, parce que son emplacement géographique correspond à une des quatre grandes zones d'upwelling et de haute productivité se trouvant entre $4-6^{\circ} ; 7-9^{\circ} ; 11-13^{\circ}$ et $14^{\circ}$ LS. (Zuta \& Guillen 1970).

10 À Huanchaco, se trouve une communauté remarquablement fière, consciente de son passé et héritière de sa culture, de ses savoirs et savoirs-faire datant de l'époque des 
Mochicas ( $\mathrm{II}^{\mathrm{e}}-\mathrm{VIII}{ }^{\mathrm{e}}$ siècles) et des Chimus ( $\mathrm{XII}^{\mathrm{e}}-\mathrm{XVI}^{\mathrm{e}}$ siècles), peuples qui étaient intimement liés à la mer. Quelques-unes de ces traditions sont restées presque invariables tout comme leurs pratiques de pêche et la culture de la totora, matière première utilisée dans la construction de leur emblématique embarcation le caballito de totora (Larco Hoyle 1938). L'archéologie nous apprend qu'aux environs de 3500 ans la totora était le matériau de construction de bateaux, probablement de "caballitos " à Huanchaco, la plus ancienne jusqu'à nos jours (Campusano 2014).

\section{«El caballito de totora » : une embarcation singulière}

Le caballito de totora est une embarcation construite avec une plante (roseau), la totora (Schoenoplectus californicus) employée depuis l'époque pré-inca pour la construction de l'embarcation appelée par les espagnols caballito de totora et qui en langue muchik était connue comme "Tup » (Ñique \& Vidal 1993, Rostworowski 2005), parmi les multiples usages de cette plante. Elle est préalablement séchée grâce à l'action du soleil. Les pêcheurs artisanaux des côtes péruviennes depuis environ cinq mille ans ont construit avec beaucoup d'habileté cette embarcation individuelle presque sans y apporter de modifications notables.

Le caballito utilisé actuellement pour pêcher (un des nombreux modèles qui existaient par le passé), quand il est neuf et sec, a un poids d'environ 40 kilos. Il atteint 4 mètres de longueur et a entre 0,60 et 1 mètre de large. Quand il sort de l'eau son poids est d'environ 80 kilos. La capacité de charge d'un caballito est d'environ 100 kilos.

Pour se déplacer avec le caballito, les pêcheurs utilisent une rame, qu'ils fabriquent euxmêmes à l'aide d'une plante appelée caña de Guayaquil Guadua angustifolia, plante semblable au bambou, provenant principalement de l'Équateur (Manual técnico del Bambú 2017).

El caballito de totora est le plus important attrait spécifique de Huanchaco et incarne également son symbole identitaire. Patrimoine culturel national du Pérou (RD № 648 INC), il symbolise une des pratiques de pêche la plus singulière au monde dont l'histoire remonte jusqu'à cinq mille ans selon les études des vestiges de la céramique des cultures préincas qui représentent la pêche en caballito de totora, études menées par Larco Hoyle, Buse de la Guerra, Lumbreras et autres archéologues. (Larco Hoyle 1938, Tramontana 2008).

\section{La construction d'un caballito de totora}

Un caballito est composé de deux faisceaux (ou bastones) qui sont attachés, composés de deux parties appelées "la mère » et "le fils ». La mère entoure le fils qui devient en quelque sorte le noyau du bateau (Figure 1). 
Figure 1 : Schéma montrant les parties et composantes d'un caballito de totora

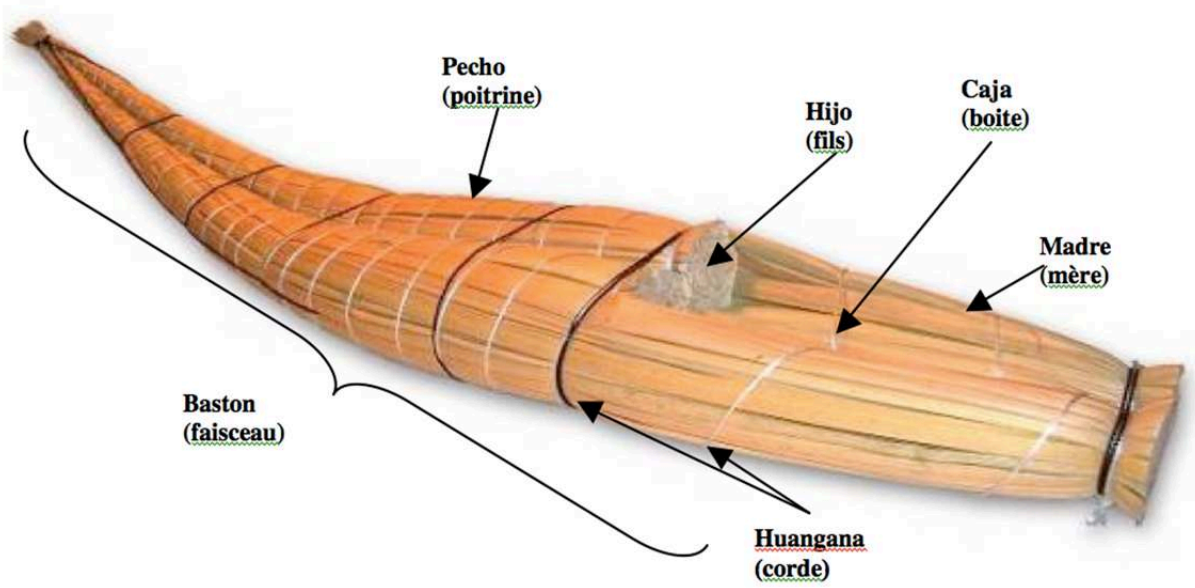

Depuis les années 80, est apparue une variation dans la construction du caballito dans le but d'économiser la totora. Cette variation consiste dans l'inclusion au centre du bateau (dans les fils) d'un noyau qui peut être fait de bouteilles en plastique ou de polystyrène sous diverses formes (Figure 2). Cette recommandation a été conseillée par les chercheurs en biologie de la pêche de l'université de Trujillo, Pérou. (Alvaro Tresierra, comm. pers.).

Figure 2 : Variations dans la construction d'un caballito. A : Caballito avec du plastique dans « Les fils ». B : Nouvelle modification du caballito avec du plastique dans « les fils » et «les mères »

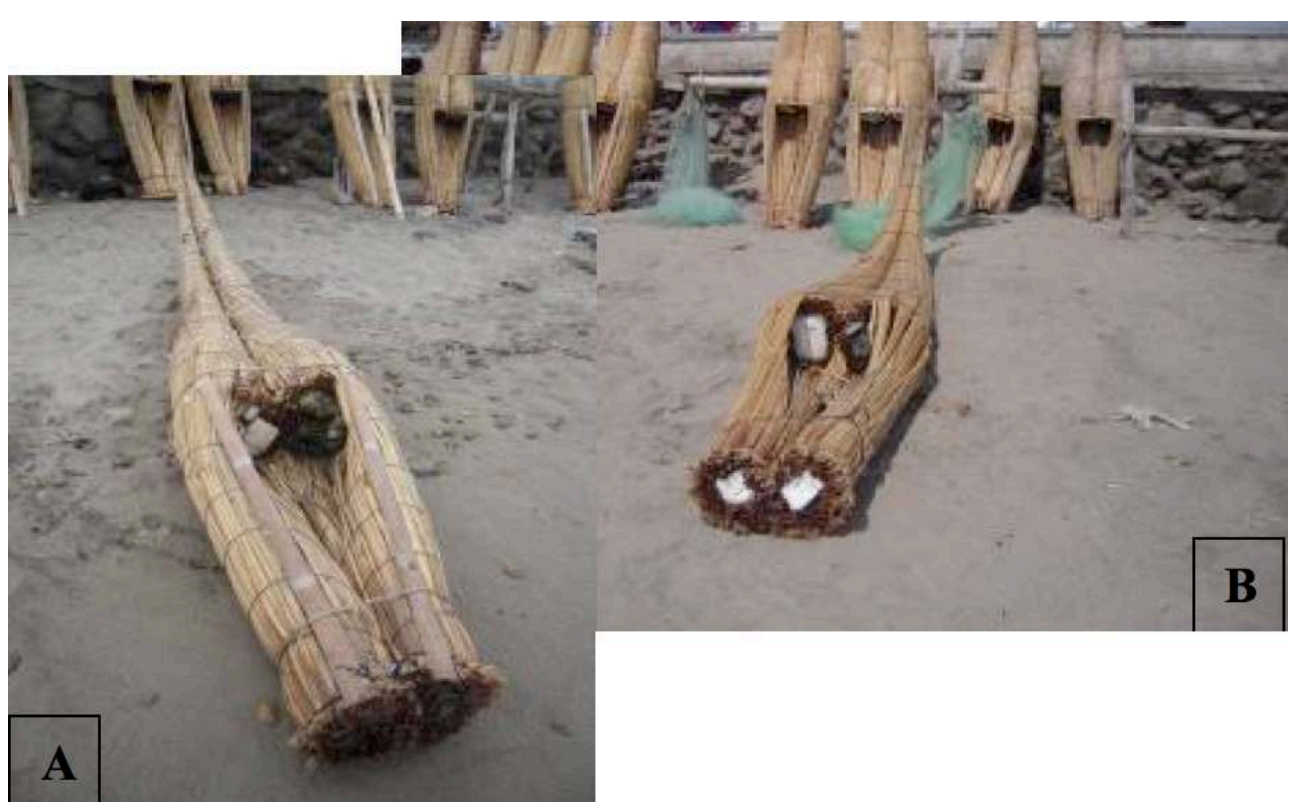

\section{Étapes à suivre dans le processus de construction}

La construction d'un caballito de totora se divise en trois étapes bien précises. 


\section{La préparation du matériau de base : la totora} puñados. Un puñado (poignée) se mesure en réunissant les deux mains pour former un cercle (Figure 3.4). Pour un caballito avec un noyau en plastique, on compte de même sept puñados pour la mère et seulement sept ou six pour le fils en fonction de la largeur souhaitée. On sépare alors en quatre groupes la totora pour chaque composant (Figure 3.5).

Figure 3 : Préparation de la totora

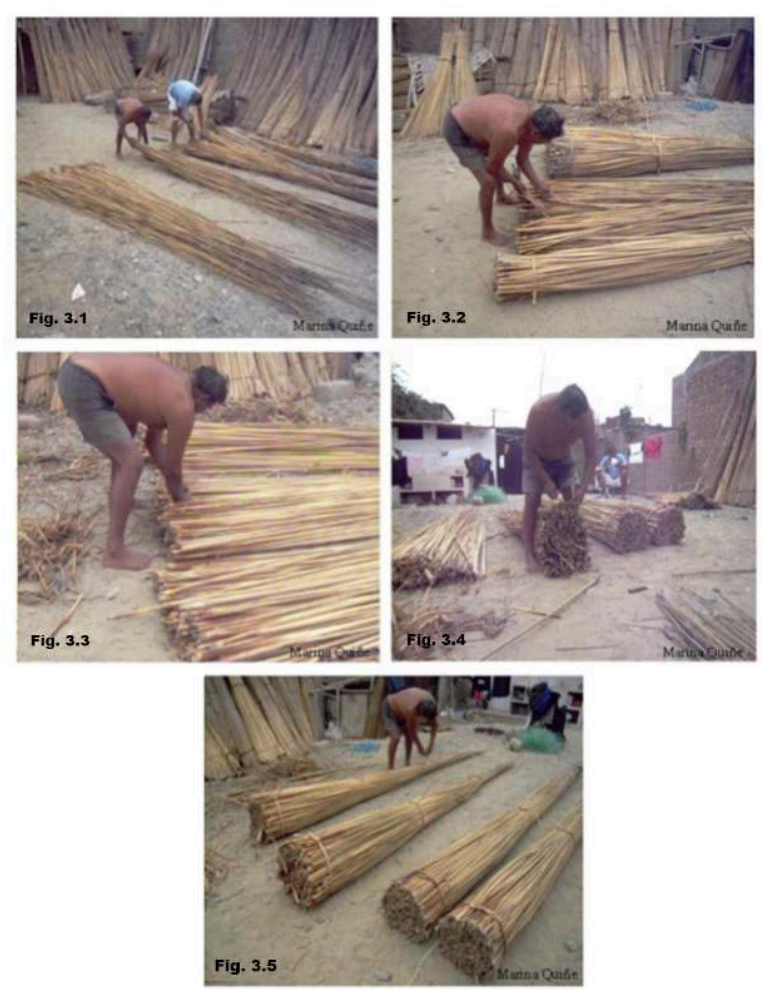

\section{Agencement des faisceaux de totora ou l'union mère-fils}

Après avoir préparé et séparé la totora à utiliser, on vérifie que les deux mères soient égales, en mesurant à l'aide de la corde qui les attachera dans l'extrémité la plus large (Figures 4.1 et 4.2). Ensuite on prend une des mères que l'on étend par terre comme un éventail, en essayant de distribuer de façon homogène toutes les tiges (Figures 4.3 et 4.4). 
23 L'étape suivante consiste à poser le fils sur la mère (Figure 4.5), en laissant dans la longueur une marge entre le bord du fils et le bord de la mère d'environ un mètre, mesurée avec une tige de totora (Figures 4.6 et 4.7). Conserver une marge égale pour les deux faisceaux est très important, car cet espace va devenir le coffre de charge du bateau, où les fils (l'un à côté de l'autre) vont définir la paroi intérieure, laquelle doit avoir une surface homogène.

Après, on s'assoit sur le fils et en s'aidant avec les genoux on commence à couvrir les flancs du fils par la mère (Figure 4.8). On sécurise l'union, en les attachant en différents points avec des feuilles de totora (Figure 4.9). On observe maintenant que le faisceau a déjà pris la forme allongée pointue de la moitié d'un caballito (Figure 4.10).

Pour fixer la forme qui deviendra une moitié du caballito, il faut commencer à attacher les deux parties qui forment le faisceau avec une corde ou huangana ${ }^{3}$. On commence par l'extrémité large de la mère, en ajustant avec deux ou trois tours de la corde bien serrés (Figure 4.11). Puis on tourne la corde tout autour du faisceau en montant vers la pointe (Figures 4.12 et 4.13). Il faut imprimer de la force mais pas en excès, et pour finir, il faut tirer sur chaque tour de la corde pour bien serrer le tout.

26 En même temps que l'on attache les faisceaux, il faut aussi donner la courbure (vers le haut) de la « poitrine » ou pecho del caballito, en appuyant doucement du centre vers la pointe, tandis que l'on fait des tours avec la corde (Figure 4.14).

27 La façon de tourner la corde autour de chaque faisceau n'est pas la même, elle se fait en sens opposé, sur un des faisceaux vers la droite (Figure 4.11) et sur l'autre vers la gauche (Figure 4.15). Quand on commence à faire le deuxième faisceau, il faut d'abord attacher la partie où commence le fils, car il faut vérifier, en mettant un faisceau à côté de l'autre, que la position des deux fils soit la même (Figure 4.16). Après ce premier passage de la corde autour de chaque faisceau, il faut tendre au maximum la corde pour éviter que l'eau ne pénètre (Figure 4.17). Au moment de finir la fixation de chaque faisceau, ils présenteront une forme similaire à celle des défenses d'un éléphant (Figure 4.18). 
Figure 4 : Agencement des faisceaux de totora

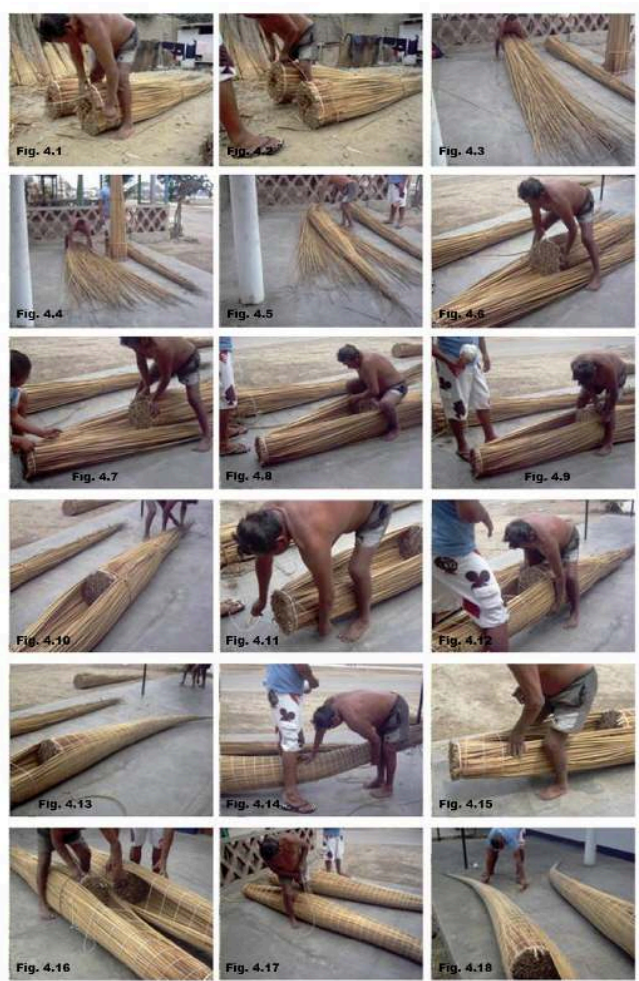

\section{L'assemblage du caballito}


Figure 5 : Assemblage d'un caballito

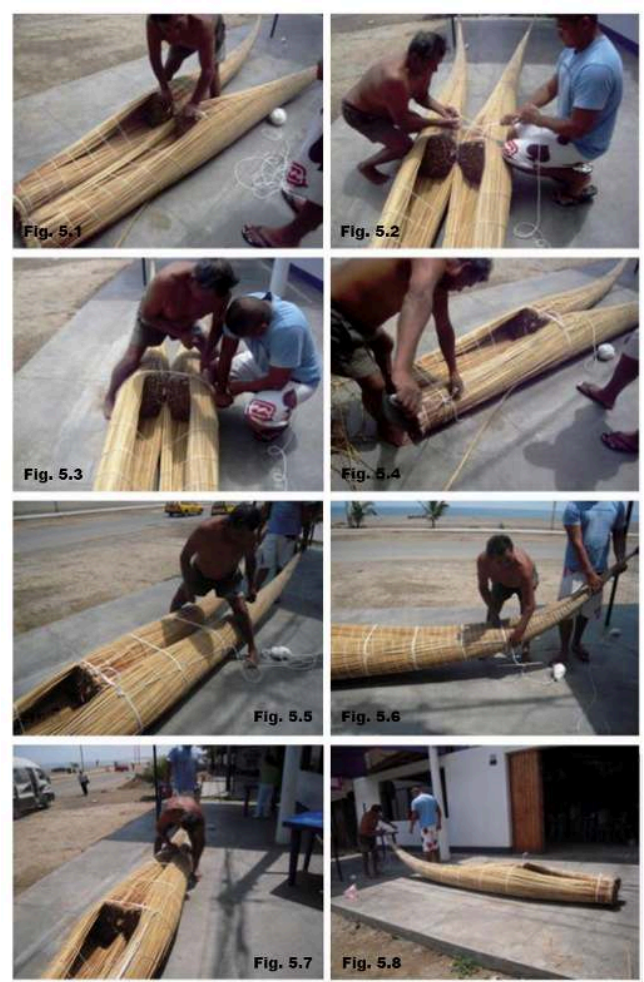

\section{La pêche en caballito de totora}

Depuis son origine, la pêche en caballito de totora s'est pratiquée de façon ininterrompue sur la côte péruvienne. Du passé préhispanique, on garde les représentations artistiques en céramique et les dessins des scènes de pêche. Avec beaucoup de détails et de symbolisme, elles représentent des pêcheurs ou des déités sur les bateaux, pour illustrer la pratique de la pêche à l'époque; elles nous montrent même dans certains cas, les espèces pêchées, espèces qui, en grande partie, continuent à être pêchées de nos jours (Larco Hoyle 1938).

33 À l'époque de la colonisation espagnole depuis le $\mathrm{xvI}^{\mathrm{e}}$ siècle, les chroniqueurs qui ont décrit les pratiques de pêche en caballito, indiquent deux façons de pêcher. La première avec une ligne et des hameçons qui constituait une pêche solitaire dans laquelle le pêcheur partait seul en mer avec sa ligne, ses hameçons, ses appâts, son cal cal (ou sac pour mettre les poissons) et une sorte d'ancre de pierre. La seconde se pratiquait avec un filet maillant de forme triangulaire (comme un sac de neuf mètres de long) appelé incaica, manipulé par deux pêcheurs, chacun sur son caballito ayant un rôle différent au moment de la pêche (Rostworowski 2005), description qui ressemble à la pêche au chalut de fond, rarement pratiquée aujourd'hui au Pérou.

Actuellement les pratiques de pêche tendent en général à minimiser les efforts, face à la rareté des ressources de la zone. C'est pour cela que le chalut de fond est devenu très rare voire a disparu. Les pêcheurs continuent de pêcher les jours où cela est possible, et parallèlement d'avoir d'autres activités qui peuvent rapporter de l'argent, ou simplement s'occupent à entretenir leurs huachaques ou zones de culture de la totora. 
Chaque pêcheur a sa zone de pêche, délimitée par des bouées attachées à des corpsmorts ou fondo, bouées qui pour un regard non averti se ressemblent toutes. Mais les pêcheurs se repèrent très bien dans la distribution des zones de pêche entre eux et visà-vis de leurs voisins de la communauté de Huanchaquito, ville face à laquelle ils vont également pêcher.

Les zones de pêche fonctionnent comme des concessions de pêche mais il n'y a aucun document ni carte, où elles seraient indiquées. L'utilisation d'une parcelle de mer, correspond donc aussi au droit coutumier à Huanchaco.

7 Avant d'aller en mer, les pêcheurs préparent leurs outils de pêche pour la journée. Ils plient et attachent d'une même corde chaque filet qui sera utilisé. S'ils vont pêcher le crabe, ils préparent sur la plage les pièges ou sacas qu'ils emporteront, en attachant à l'intérieur l'appât composé de viscères de poissons. S'ils vont à la pêche à la ligne, ils en préparent de différentes sortes en veillant à ce que l'hameçon soit correctement fixé. En guise d'appât, ils utilisent Emerita analoga, un crustacé vivant dans le sable des côtes ouest du continent américain, connu des pêcheurs sous le nom de capusa et de muy muy (deux stades différents de développement du crustacé); ils utilisent l'un ou l'autre en fonction du type de poisson recherché. On utilise aussi des leurres ou muestras pour attirer les poissons. Les leurres se font actuellement avec le métal des antennes de télévision, auparavant ils étaient fabriqués avec de l'étain et toujours polis avant d'aller en mer. Quand un pêcheur va pêcher à la ligne, il emporte une pierre attachée à une corde, en guise d'ancre, comme autrefois.

De nos jours, les principales pratiques de pêche à Huanchaco consistent en l'utilisation de filets maillants de un ou trois panneaux (trémail) et de pièges pour la pêche au crabe. Le pêcheur observe les marées pour savoir à quel moment il doit aller placer ses filets. Selon les pêcheurs, à marée montante, les poissons vont manger, c'est donc un moment favorable pour la pêche. Avec ses filets placés dans le coffre ou la caja du caballito, le pêcheur se dirige vers sa zone de pêche. Cela peut prendre 20 à 40 minutes. Arrivé sur place, il étend doucement ses filets en cherchant les cordes qui sont de chaque côté de la partie supérieure des bouées ou flotteurs.

jullet à novembre-décembre, c'est la "saison du crabe " Platyxanthus orbignii. Certains jours, les pêcheurs alternent leurs activités entre la pose des filets et celle des pièges à crabes. Comme la saison du crabe coïncide avec l'hiver, les jours de pêche diminuent considérablement. Parfois on ne pêche qu'un jour ou deux dans la semaine.

$\mathrm{Au}$ retour sur la plage, et après avoir vendu leur pêche, les pêcheurs doivent s'occuper de leur caballito; ils le dressent pour le vider de l'eau qui y est entrée, puis ils le transportent sur l'épaule vers l'endroit de la plage où tous sont rangés pour sécher jusqu'au lendemain (Figure 6). 
Figure 6 : La pêche en caballito

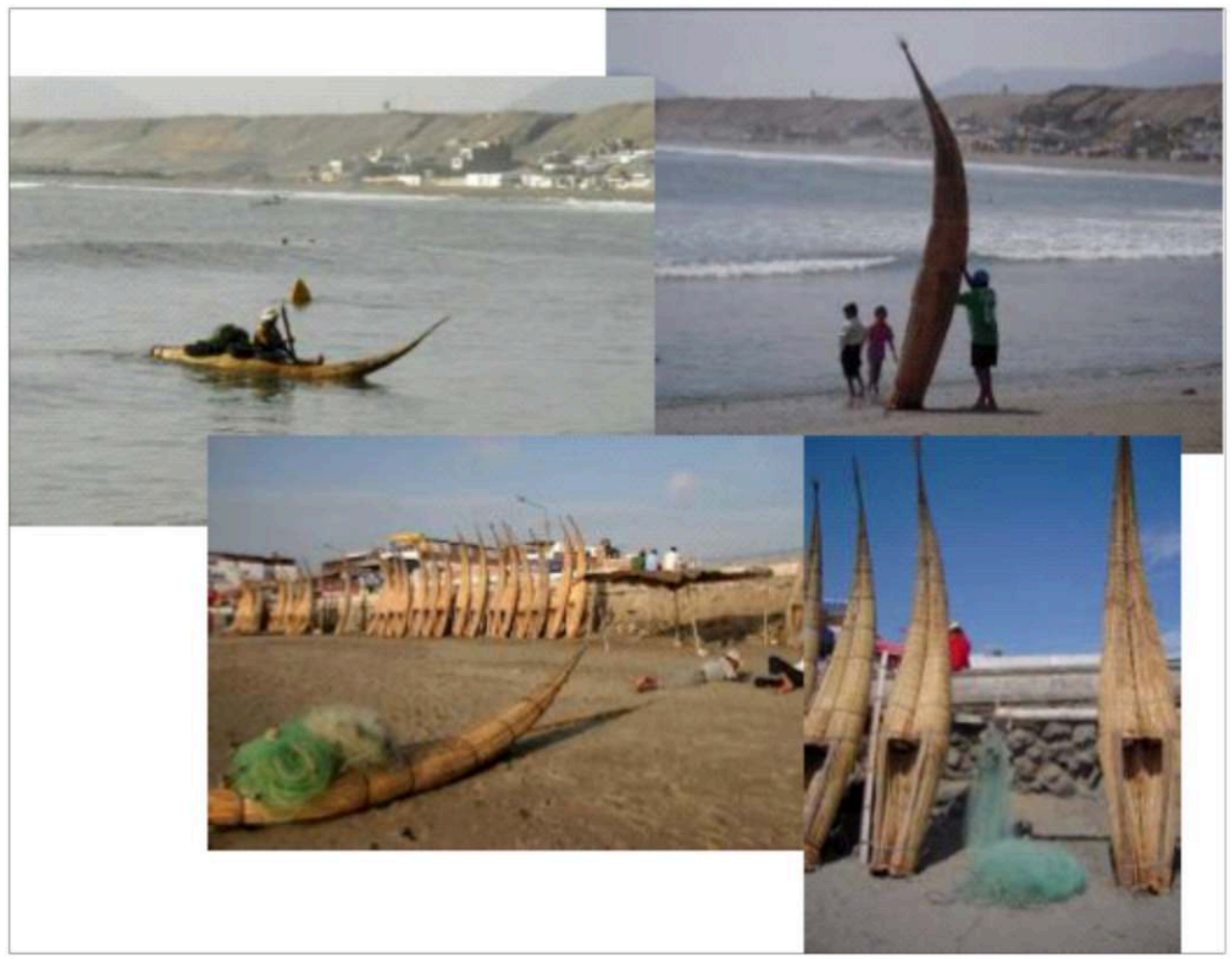

41 Sur le littoral nord péruvien, les villes de pêcheurs où continuent à se pratiquer cette pêche et où les savoirs et les savoir-faire sont encore transmis, ne sont plus que trois. Huanchaco est la seule à produire encore la matière première, qu'elle vend aux deux autres. La communauté doit faire face à une problématique complexe, à commencer par la disparition des zones de culture de totora, due à l'érosion de la mer et au manque de main d'œuvre pour le maintien et la transmission des savoirs. Cela n'est qu'un problème parmi d'autres qui affligent cette communauté, et qui affectent directement le maintien de cette pratique de pêche millénaire.

Au MNHN, au Département Hommes et Environnement, à Serge Bahuchet et au peuple de Huanchaco, particulièrement aux pêcheurs. Un grand merci à M. Fidel Huamanchumo, pêcheur de toute une vie, qui a construit le Caballito de totora qui apparaît dans cette publication et qui est exposé au Musée de l'Homme de Paris.

\section{BIBLIOGRAPHIE}

Campusano 0. 2014 - Descubren evidencia más antigua del caballito de totora. El Comercio. 08/03/2014. [En ligne :] https://elcomercio.pe/peru/la-libertad/descubren-evidencia-antiguacaballito-totora-348289?foto=1 
Carbajal W., Galan J. \& De la Cruz J. 2005 - Prospección del recurso Chondracanthus chamissoi “cochayuyo" en la playa de Huanchaco, Trujillo. Junio-julio 2005. Informe. IMARPE.

García A. 2001 - Genero en la pesca artesanal en el Perú. Investigaciones Sociales 5 (7) : 43-63.

Larco Hoyle R. 1938 - Los Mochicas. Tomo I. Lima, Perú. 333 p. [En ligne :] http:// www.museolarco.org/publicaciones.shtml

Majluf P. 2006 - El sector pesquero. (UPCH) - World Bank presentation. [En ligne :] siteresources.worldbank.org/.../Patricia_Majluf_Pesca.ppt [PPT]

Manual Técnico del Bambú (Guadua angusfolia kunth.) para productores 2017 - Universidad de Sassari - Fundación AVSI. 88 p.

Ñique M. \& Vidal J. 1993 - Conservación ex situ de Schoenoplectus californicus (C.A. Mey) Sojak, en Huanchaco (Trujillo-Perú). Antenor Orrego 2.

Perú - Ministerio de la Producción 2015 - Anuario Estadístico Pesquero y Aquícola. [En ligne :] https://www.produce.gob.pe/documentos/estadisticas/anuarios/anuario-estadisticopesca-2015.pdf

Revista Pesca 2011 - La paradoja perversa. [En ligne :] revistapesca.blogspot.com/2011/08/laparadoja-perversa.html.

Rostworowski M. 2005 - Recursos naturales renovables y pesca, siglos XVI-XVII / Curacas y sucesiones, costa norte. Obras completas: vol. IV. Lima, IEP, 330 p.

Tramontana 0. 2008 - Caballito de totora, la primera embarcación unipersonal en surcar olas del mundo. [En ligne : ] http://www.olasperu.com/articulos_display.php?id=32.

Zuta S. \& Guillen O. 1970 - Oceanografía de las Aguas Costas del Perú, Dpto. de Oceanografía. Bol. Inst. Mar Perú Callao 2 : 157-324.

\section{NOTES}

1. Cet article correspond à une partie des recherches menées dans la communauté de Huanchaco, dans le cadre de mon Master en Sciences de la Nature et de l'Homme sous la direction de Serge Bahuchet au Muséum national d'Histoire naturelle de Paris, France.

2. Par Décret Suprême №12-2001-PE, la zone comprise entre la ligne de côte et les cinq mille marins correspond à une aire exclusive pour la pêche artisanale; elle protège la zone de reproduction des ressources marines.

3. La corde de nos jours est achetée dans le commerce, elle est donc synthétique. Autrefois elle était d'origine végétale.

\section{RÉSUMÉS}

$\mathrm{Au}$ Pérou, la pêche est pratiquée depuis plus de dix mille ans et représente toujours l'une des activités économiques les plus importantes. Dans cet article, nous nous intéressons à une pratique de pêche ancestrale qui persiste dans le temps sans variations significatives : la pêche en 
caballito de totora à Huanchaco. Nous présentons le bateau support principal de cette activité, le caballito de totora, lequel a gardé une structure faisant appel à un savoir-faire pratiquement inchangé depuis plus de deux mille ans.

En el Perú, la pesca es una actividad practicada desde hace más de diez mil años y representa aún una de las actividades económicas más importantes. En éste articulo nos interesamos a una práctica de pesca ancestral que persiste en el tiempo sin variaciones significativas: la pesca en caballito de totora en Huanchaco, enfocándonos en conocer mejor la embarcación que permite que sea posible esta actividad, el caballito de totora, el cual ha mantenido una estructura y fabricación artesanal básicamente sin cambios desde hace mas de dos mil años.

In Peru, fishing has been practiced for more than ten thousand years and still represents one of the most important economic activities. In this paper we are interested in an ancestral fishing practice that persists in time without any significant variation: fish riding on a boat called caballito de totora, in Huanchaco, Peru. We focus on acquiring better knowledge about the boat, el caballito de totora, which has maintained its structure and craftsmanship for more than two thousand years.

\section{INDEX}

Mots-clés : savoir-faire, bateau, pêche artisanale, techniques de pêche Index géographique: Huanchaco, Pérou

Keywords : know how, artisanal fishing boat, fishing technique, Huanchaco, Peru

Palabras claves : saber hacer, bote de pesca artesanal, técnicas de pesca, Huanchaco, Perú

\section{AUTEUR}

\section{MARINA QUIÑE}

PSL Research University: EPHE-UPVD-CNRS, USR3278 CRIOBE, 66860 Perpignan, France

marina.quine@gmail.com 